\title{
The Activation of DNA Damage Response is the Basis of the Occurrence, Development and Resistance to Radio- or Chemotherapy in Ovarian Cancer
}

\author{
Changsheng Peng ${ }^{1 *}$ and Wei Zhang ${ }^{2}$ \\ ${ }^{1}$ Department of Gynecology and Obstetrics, West China Second University Hospital, China \\ ${ }^{2}$ Key Laboratory of Birth Defects and Related Diseases of Women and Children (Sichuan University), Ministry of Education, China \\ *Corresponding author: Changsheng Peng, Department of Gynecology and Obstetrics, West China Second University Hospital, \\ Sichuan University, Chengdu, China.
}

To Cite This Article: Changsheng Peng, Wei Zhang, The Activation of DNA Damage Response is the Basis of the Occurrence, Development and Resistance to Radio- or Chemotherapy in Ovarian Cancer. 2020 - 11(3). AJBSR.MS.ID.001622. DOI: 10.34297/AJBSR.2020.11.001622.

Received: 制 December 13, 2020; Published: 阱 December 16, 2020

\section{Opinion}

Genome surveillance system is critical for eukaryotic cells to maintain genome stability [1,2]. Cell cycle checkpoints is quickly activated upon detecting various forms of DNA lesions, ensued by loading of repair factors to eliminate DNA damage [3]. ATM (Ataxia-Telangiectasia mutated) and ATR (ATM and Rad 3-related), the upstream checkpoint kinase, initiate damage detection and signal transduction after exposure to genotoxic insults including ionizing radiation or chemotherapeutic reagents [4]. To eliminate DNA lesions generated during genomic replication, ATR-dependent replication stress is activated to modulates the activities of the cell cycle regulator to halt cell cycle. In addition, ATR phosphorylates CHK1 to stall and thus maintain the integrity of replication fork, preventing catastrophe caused by fork collapse [5-6]. This is particularly important for the replication of fragile structures such as nucleolus-localized rDNA sequence. It is well known that ovarian cells have been exposed to high estrogen environment (some labs estimated the estrogen concentration in ovary is 100times higher than other tissues [7] for a long time, which greatly induces genomic instability and leads to ovarian cancer [8]. Our study found that compared with normal ovarian cells, the genome monitoring system of ovarian cancer cells still plays a role. Cancer cells rely on robust DNA damage response activation to survive the harsh environment, such as genotoxicity-based radio- or chemotherapy. In addition, we also found that irradiating (IR) tumor cells with $\mathrm{x}$-rays or adding chemotherapeutic drugs (cisplatin) could not only kill most ovarian tumor cells, but also promote tumor metastasis. Other laboratories have also reported that radiotherapy and chemotherapy may promote the metastasis of breast and prostate cancer by $[9,10]$. This is because radiotherapy and chemotherapy break the genetic stability of tumor cells and promote their epithelial-mesenchymal transformation. The effect of tumor metastasis was significantly weakened after we added ATM inhibitor (ATMi) or ATR inhibitor (siATR) to the cells.

\section{Conclusion}

In conclusion, we believe that radiotherapy and chemotherapy can promote ovarian cancer metastasis, there is hope to find the relevant Drug action targets, combined with radiotherapy and chemotherapy to completely kill tumor cells, which may be a good research idea.

\section{Conflict of Interest}

There is no conflict of interest.

\section{References}

1 Gorgoulis VG, Vassiliou LV, Karakaidos P, Panayotis Z, Athanassios K, et al. (2005) Activation of the DNA damage checkpoint and genomic instability in human precancerous lesions. Nature 434(7035): 907-913.

2 Negrini S, Gorgoulis VG, Halazonetis TD (2010) Genomic instability - an evolving hallmark of cancer. Nat Rev Mol Cell Biol 11(3): 220-228.

3 Shiloh Y, Ziv Y (2013) The ATM protein kinase: regulating the cellular response to genotoxic stress, and more. Nature Reviews Molecular Cell Biology 14(4): 197-210.

4 Song L., Tang Z., Peng C, Yang Y, Guo C, et al. (2020) Cell type-specific genotoxicity in estrogen-exposed ovarian and fallopian epithelium. BMC Cancer 20(1): 1020.

5 Blackford AN, Jackson SP (2017) ATM, ATR, and DNA-PK: The Trinity at the Heart of the DNA Damage Response. Mol Cell 66(6): 801-817. 
6 Nakamura Y (1998) ATM: the p53 booster. Nat Med 4(11): 1231-1232.

7 Lindgren PR, Backstrom T, Cajander S, Damber MG, Olofsson JI (2002) The pattern of estradiol and progesterone differs in serum and tissue of benign and malignant ovarian tumors. International Journal of Oncology 21(3): 583-589.

8 Feys L, Descamps B, Vanhove C, Anne V, Liv V, et al. (2015) Radiationinduced lung damage promotes breast cancer lung-metastasis through CXCR4 signaling. Oncotarget 6(29): 26615-26632.
9 Mi S, Qu Y, Chen X, Zhihua W, Pengxiang C, et al. (2020) Radiotherapy Increases 12-LOX and CCL5 Levels in Esophageal Cancer Cells and Promotes Cancer Metastasis via THP-1-Derived Macrophages. Onco Targets Ther 13: 7719-7733.

10 Lee SY, Jeong EK, Ju MK, Hyun MJ, Min YK, et al. (2017) Induction of metastasis, cancer stem cell phenotype, and oncogenic metabolism in cancer cells by ionizing radiation. Mol Cancer 16(1): 10 . 